\title{
Numerical analysis of Separate Vortex in the Diffuser
}

\author{
Yong-rui Sun ${ }^{a}$, Chuan-lei Yang ${ }^{b}$, He-chun Wang ${ }^{c}$ Xiang-huan $\mathrm{Zu}{ }^{d}$
}

Scool of Power and Energy Engineering, Harbin Engineering University, Harbin 150001, China

asyr1988@hrbeu.edu.cn, ${ }^{b}$ wanghechun@hrbeu.edu.cn,

cyangchuanlei@hrbeu.edu.cn, ${ }^{\mathrm{c}}$ zuxianghua@hrbeu.edu.cn

Keywords: centrifugal compressor; vaneless diffuser; unstablity mechanism; casing structure.

\begin{abstract}
In order to simulate the flow structure accurately and find out the inducement of unstability in a centrifugal compressor with casing structure, three different cases of models were established. By contrasting the simulation result of Case1 and Case2 with experiment data, the accuracy of grids model and turbulence model was validated. Compared with utilized a "Mixing Plane" at the Rotor-Stator interface in Case1 and Case2, the flow in diffuser of Case 3 was solved in the rotating reference frame. Compared simulation result with Case1 and Case2, the accuracy of Case3 model was validated. By studying the flow structure in diffuser, the fusion and development of the ISV result in the scope of backflow extending to blade passage, which is inducement of compressor instability. A tracing back analysis was carried out with Case 3 model to investigate the formation of ISV. The blade leakage flows and strong pressure variations at outlet of blade passage were the cause of ISV.
\end{abstract}

\section{Introduction}

Although considerable work had been expended over past years, the mechanism of instability didn't reach an agreement especially for centrifugal compressor. Hagelstein carried out researches on influences of single component or components interactions ${ }^{[1]}$. Based on the methods of numerical and experiment, the analysis of flow characteristics was conducted abroad ${ }^{[2,3,4]}$. Since the simple geometry of vaneless diffuser, it's the most favorite one to be studied. There were some explanations about the inducement of instability in centrifugal compressor, which were boundary layer separation, core flow separation, or even the mutual interaction ${ }^{[5]}$. Marsaner found a boundary layer separation remains downstream of the suction slot and is due to unsteadiness, namely the scrolling of the high pressure waves which generates intense instantaneous adverse pressure gradients by unsteady simulation ${ }^{[6]}$. Recently with continuous increases in computational power and advances in CCD sensor technology, DPIV provides near real-time flow field measurements. Guo qiang and Ohuchina found the a boundary separation at the both shroud and hub of vaneless diffuser in rotating stall condition by the measurements of DPIV ${ }^{[7,8]}$. Their researches showed the rotating stall mechanism of the vaneless diffuser compressor should have a significant relationship with the separation in diffuser. But the formation and the development of separation in diffuser was not reported. And the relationship of the flow in impeller and diffuser was not mentioned.

\section{Computing method}

Compressor Parameters. The test compressor is a vaneless diffuser centrifugal compressor with casing structure, the details are showed in fig. 1.

Computation Grids and Boundary Conditions. A composite grid system is used to simulate the detail flow in compressor. Figure 2 shows the computation grid. ANSYS CFX was used to carry out the numerical study. The averaged static pressure was specified at outlet boundary. The compressor is simulated under two rotating speeds: $500 \mathrm{r} / \mathrm{s}(\mathrm{Mu}=0.953)$ and $700 \mathrm{r} / \mathrm{s}(\mathrm{Mu}=1.33)$.In Ref[5,6], because of the limitation of experiment, the test compressor did install a volute. So in order to simulation flow in compressor under the same condition, no volute is in the model of Case 2. Mixing Plane is applied in the interface of the rotor and stator for the first two cases. An article showed the weakness of the 
Mixing Plan ${ }^{[8]}$. So in the model of Case 3, all of the impeller and diffuser are set as rotating domain (named Overall Rotor model), meanwhile the grid of Case 3 is same as the grid of Case 2. By contrasting the simulation result of different cases, the effect of Mixing Plan to predict flow in compressor is clear.

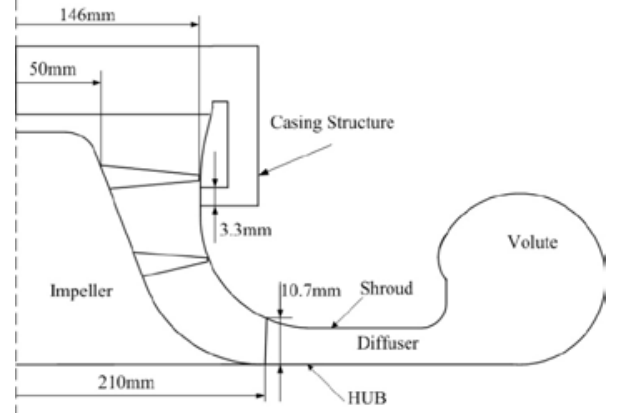

Figure 1 Schematic view of the compressor

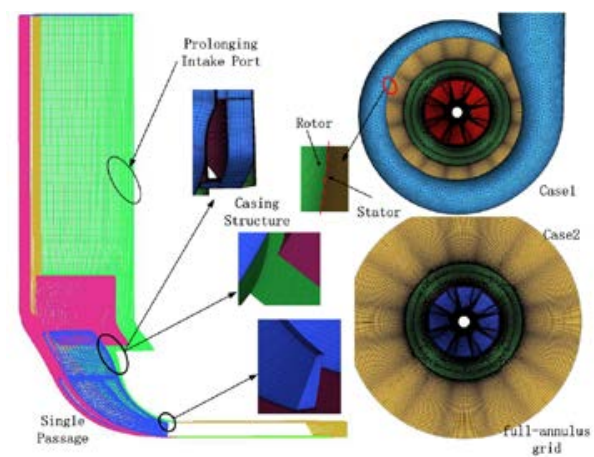

Figure 2 Created mesh of the Compressor

Validation of the Numerical Results. To validate the numerical model, the performance of the compressor is simulated under two rotating speeds: $\mathrm{Mu}=0.95, \mathrm{Mu}=1.33$. Under these two speeds, the fan was operating under typical transonic and subsonic conditions respectively. The simulation's performance was compared with the experiment's performance, as shown by Figure 3. Under the two rotating speeds, the trends of the experiment performance curves were finely repeated by the simulations of Case 1. The simulation pressure rate of Case 2 and Case 3 is higher than that of Case 1 . Because of no volute in the model of Case 2, the flow losses in Case 2 is lower than that in Case 1. In Case3, due to no mix plane in the model, there is a small difference in characteristic line between Case2 and Case3. Contours of absolute tangential velocities at 105\% impeller exit radius of different case are shown as Figure 4, the (a), (b), (c) respectively represent result of Case1, Case 2 and Case3. In figure 4, the distribution of Vt is nearly the same. Although there is a small difference in characteristic line, the simulation result of Case 3 can be used to carry out a qualitative analysis for the flow structure

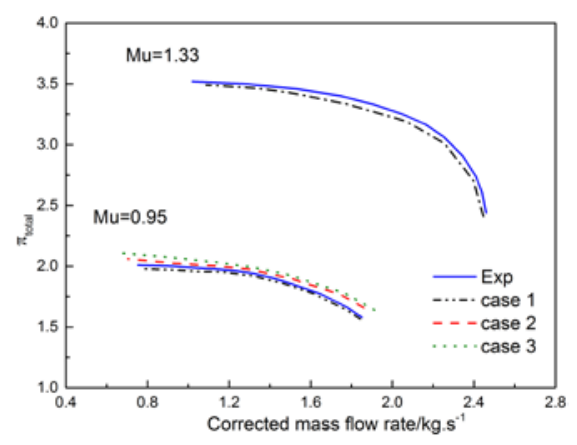

Figure 3 Performance of the compressor under two rotating speeds: $\mathrm{Mu}=0.95, \mathrm{Mu}=1.33$

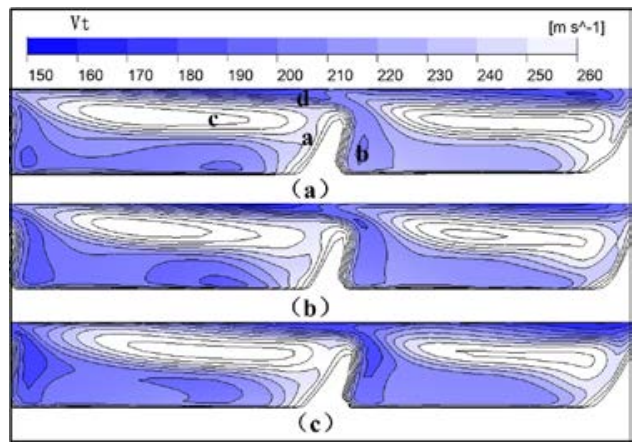

Figure 4 Contours of absolute tangential velocities at $105 \%$ impeller exit radius of different case

Results and Discussions. In order to analyze the flow of boundary layer in the diffuser, Fig 5 compares the flow structure in diffuser in different cases from the chock point to numerical stall point. To validate the calculated model, the flow structure in diffuser at numerical point of Case 2 is compared with the result in Ref[8]. The flow structures of two results are shape of S. That means the prediction of separation is accurate. At all the operation, several separation vortexes of boundary layer always appear at the shroud of diffuser in all the cases. So the separation vortexes are caused by the flow property of compressor, and of course the appearance of vortexes is not the inducement of instability. The vortexes here are named Initial Separate Vortexes (ISV).With the mass flow decreasing from chock point, the vortexes gather and fuse at the exit of impeller, which result in the scope of backflow extending (blue line in Fig. 5). It should be the inducement of instability, the effective flow area at the exit of impeller is reduced by the large scope of backflow. 
In Fig.5, the distribution and development of ISV in three cases is similar. Compared with the ISV in Case 3, it's clear that the Mixing plane cuts the vortex into two parts at the position of A. So adopting the method of "Mixing Plane" would bring in the calculation error at the place of "Mixing Plane", and the Overall Rotor model could avoid the error. Since the work of volute in Case 1, there're no vortexes at the hub of diffuser contrast with the flow in Case 2 and Case 3 near the stall point. These vortexes are named Second Separation Vortex. Because the accuracy of flow structure has already proved, accuracy is poor when Second Separate Vortex is simulated. For a general compressor with a volute, it can be found the scope of back flow enlarge to diffuser at the decreasing of mass flow. This may be a possible inducement of instability. As adopting Overall Rotor model can avoid the calculation error at Mixing Plan, and meanwhile it's easier to analyze the flow in the rotating reference frame, so the detail flow analysis is carried on with the model of Case 3. It can be noticed the ISV contains two parts: ISV I and ISV II, as shown in Fig. 5.

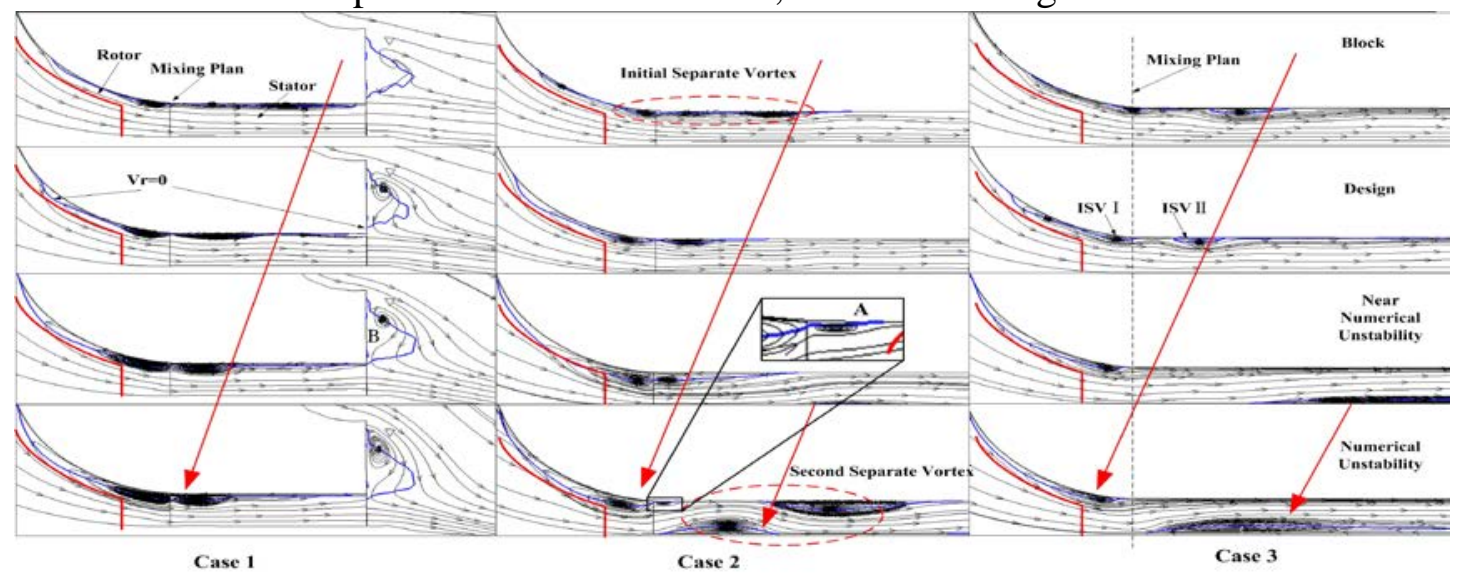

Figure 5 flow structure in diffuser in different cases from the chock point to numerical stall point

Detail Flow Analysis. In Fig.4, the flow feature can be mainly divided into four parts, marked as "a", "b”, “c” and "d". There're considerable improvements in understanding of the flow features are obtained by tracing back the origins of the diffuser flow patterns inside the impeller. For this purpose, the flow streamlines are used to visualize the diffuser flow alongside the impeller flow in the rotating frame of reference, as shown in Fig.6.

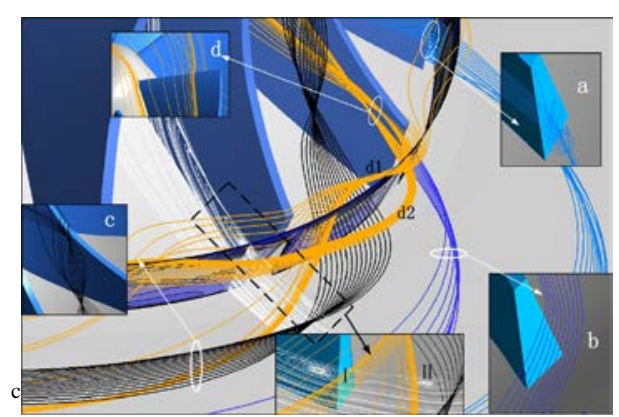

Figure 6 Detail flow analysis in compressor

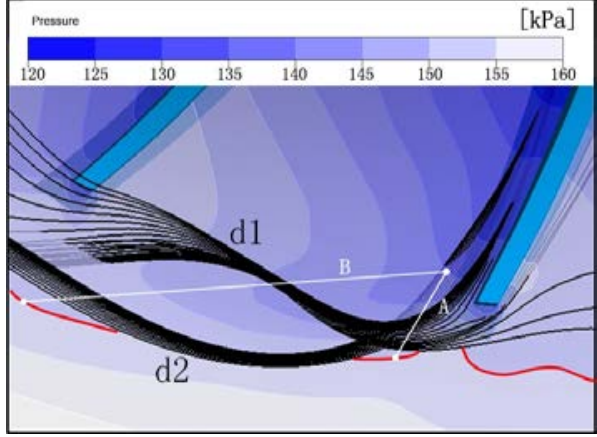

Figure 7 Flow streamlines in conjunction with the contours of static pressure at $70 \%$ of the diffuser height

In Figure 6, it can be found that region "a" and "b" is formed from the impeller trailing edge wake, and it's dissipated quickly downstream of the trailing edge. In consideration of rotating direction, the low tangential velocity patch next to the trailing edge wake (region "b") may be generated by effect of the pressure surface flow near the trailing edge.

The streamlines are released in the region c of high tangential velocity, and are traced back in the impeller. Inside the diffuser, the streamlines are shown in the relative frame of reference, so they can be linked to the flow inside the impeller. It can be observed that region "c" is formed mainly of the impeller tip leakage flows and the flow from blade suction, and which turns and distorts in diffuser, and finally this part flow become the mainly component flow of ISV II. The flow from blade suction is originating at root of blade, which is raised to the tip of bade by the impact of centrifugal force. So 
this part flow is called suction raised flow. The nature of the flow in the low tangential velocity region $\mathrm{d}(\mathrm{d} 1, \mathrm{~d} 2)$ near the diffuser casing is also complex, and the flow from region $\mathrm{d}$ can be divided into two parts: d1, d2, as shown in Fig.6. It can be observed that this region originates from suction raised flow and the reverse flow from other passage, which turns rapidly towards the casing and pressure surface of next blade after entering the vaneless diffuser. The flow from region $\mathrm{d} 1$ and $\mathrm{d} 2$ becomes the mainly component flow of ISV I , ISV II . Since the gather and fusion of ISV is the inducement of instability, and the flow coming from region c and region d is main part of ISV. So with the mass flow decreasing, the development of the impeller tip leakage flows and suction raised flow result in instability of compressor.

As shown in the Fig. 7, the flow from region c and region d turning rapidly at exit of impeller would be formation of ISV. To demonstrate the causes of this phenomenon, the flow streamlines are shown in conjunction with the contours of static pressure at $70 \%$ of the diffuser height in Fig. 8. Strong pressure variations downstream of the impeller trailing edge can be observed in the figure. Line $\mathrm{A}$ and Line $\mathrm{B}$ express the same pressure variation. Compared with Line $\mathrm{B}$, the pressure rises rapidly along direction of Line A. So the flow here reverses to the impeller and changes the flow direction along direction which pressure rises slowly.

\section{Summary}

Adopting the method of "Mixing Plane" would bring in the calculation error at the place of "Mixing Plane", and the Overall Rotor model could avoid the error. But the accuracy is poor when Second Separate Vortex is simulated.

With the mass flow decreasing, the development of the impeller tip leakage flows and suction raised flow, which are the nature of region $\mathrm{c}$ and region $\mathrm{d}$, result in vortexes gathering and fusing at the exit of impeller and the scope of backflow enlarging. The effective flow area at the exit of impeller is reduced by the large scope of backflow, and it's the inducement of compressor. A tracing back analysis was carried out with Case 3 model to investigate the formation of ISV. The blade leakage flows and strong pressure variations at outlet of blade passage were the cause of ISV.

\section{References}

[1] Hunziker R. The Operational Stability of a Centrifugal Compressor and its Dependence on the Characteristics of the Subcomponents[J]. Journal of turbomachinery, 1994, 116(2): 250-259.

[2] Bright, M. Investigation of pre-stall mode and pip inception in high speed compressors through the use of correlation integral. American Society of Mechanical Engineers.

[3] Wernet, M. P., Bright, M. M., \& Skoch, G. J. (2001). An investigation of surge in a high-speed centrifugal compressor using digital PIV. Journal of turbomachinery, 123(2), 418-42

[4] Lin, F., Chen, J., \& Li, M. (2002). Practical issues of wavelet analysis of unsteady rotor tip flows in compressors. AIAA, 4082, 2002.

[5] Gao, C. Analysis of geometries' effects on rotating stall in vaneless diffuser with Wavelet Neural Networks.International journal of rotating machinery, 2007.

[6] Marsan, A. Influence of Unsteadiness on the Control of a Hub-Corner Separation Within a Radial Vaned Diffuser. Journal of Turbomachinery, 137(2), 021008.

[7] Guo Q. Experiment and numerical investigation on stall flow phenomenon in the centrifugal compressor with vaneless diffuser. Ph.D. thesis, Shanghai Jiao Tong University. 48-50.

[8] Ohuchida. Experimental Study of Rotating Stall in Vaneless Diffuser of a Centrifugal Compressor. American Society of Mechanical Engineers. 